\title{
The Parameterized Suffix Tray
}

\author{
Noriki Fujisato $^{1}$, Yuto Nakashima ${ }^{1}$, Shunsuke Inenaga ${ }^{1,2}$ Hideo Bannai $^{3}$, and \\ Masayuki Takeda ${ }^{1}$ \\ ${ }^{1}$ Department of Informatics, Kyushu University, Fukuoka, Japan \\ \{noriki.fujisato, yuto.nakashima, inenaga, takeda\}@inf.kyushu-u.ac.jp \\ ${ }^{2}$ PRESTO, Japan Science and Technology Agency, Kawaguchi, Japan \\ ${ }^{3}$ MESD Data Science Center, Tokyo Medical and Dental University, Tokyo, Japan \\ hdbn.dsc@tmd.ac.jp
}

\begin{abstract}
Let $\Sigma$ and $\Pi$ be disjoint alphabets, respectively called the static alphabet and the parameterized alphabet. Two strings $x$ and $y$ over $\Sigma \cup \Pi$ of equal length are said to parameterized match ( $p$-match) if there exists a renaming bijection $f$ on $\Sigma$ and $\Pi$ which is identity on $\Sigma$ and maps the characters of $x$ to those of $y$ so that the two strings become identical. The indexing version of the problem of finding p-matching occurrences of a given pattern in the text is a well-studied topic in string matching. In this paper, we present a state-of-the-art indexing structure for p-matching called the parameterized suffix tray of an input text $T$, denoted by PSTray $(T)$. We show that PSTray $(T)$ occupies $O(n)$ space and supports pattern matching queries in $O(m+\log (\sigma+\pi)+o c c)$ time, where $n$ is the length of $t, m$ is the length of a query pattern $P, \pi$ is the number of distinct symbols of $|\Pi|$ in $T, \sigma$ is the number of distinct symbols of $|\Sigma|$ in $T$ and occ is the number of p-matching occurrences of $P$ in $T$. We also present how to build $\operatorname{PSTray}(T)$ in $O(n)$ time from the parameterized suffix tree of $T$.
\end{abstract}

\section{Introduction}

Parameterized Pattern Matching (PPM), first introduced by Baker [3] in 1990's, is a wellstudied class of pattern matching motivated by plagiarism detection, software maintenance, and RNA structural matching [3, 24, 20].

PPM is defined as follows: Let $\Sigma$ and $\Pi$ be disjoint alphabets. Two equal-length strings $x$ and $y$ from $\Sigma \cup \Pi$ are said to parameterized match (p-match) if $x$ can be transformed to $y$ by applying a bijection which renames the elements of $\Pi$ in $x$ (the elements of $\Sigma$ in $x$ must remain unchanged). PPM is to report every substring in a text $T$ that p-matches a pattern $P$.

In particular, the indexing version of PPM, where the task is to preprocess an input text string $T$ so that parameterized occurrences of $P$ in $T$ can be reported quickly, has attracted much attention for more than two decades since the seminal paper by Baker [3].

Basically, the existing indexing structures for p-matching are designed upon indexing structure for exact pattern matching. Namely, parameterized suffix trees [3], parameterized suffix arrays [8, parameterized DAWGs [21], parameterized CDAWGs [21, parameterized 
position heaps [18, 11, and parameterized BWTs [14] are based on their exact matching counterparts: suffix trees [25], suffix arrays [19], DAWGs [4], CDAWGs [5], position heaps [18, 9], and BWTs [6], respectively. It should be emphasized that extending exactmatching indexing structures to parameterized matching is not straightforward and poses algorithmic challenges. Let $n, m, \pi$ and $\sigma$ be the lengths of a text $T$, a pattern $P$, the number of distinct symbols of $|\Pi|$ that appear in $T$ and the number of distinct symbols of $|\Sigma|$ that appear in $T$, respectively. While there exist a number of algorithms which construct the suffix array for $T$ in $O(n)$ time in the case of integer alphabets of polynomial size in $n$ [10, 15, 17, 16, 22, 1, the best known algorithms build the parameterized suffix array for $T$ (denoted PSA $(T))$ in $O(n \log (\sigma+\pi))$ time via the suffix tree [3, 24, or directly in $O(n \pi)$ time [12. The existence of a pure linear-time algorithm for building $\operatorname{PSA}(T)$ and the parameterized suffix tree (denoted PSTree $(T)$ ) in the case of integer alphabets remains open.

PPM queries can be supported in $O(m+\log n+o c c)$ time by PSA $(T)$ coupled with the parameterized LCP array (denoted $\operatorname{PLCP}(T))$ [8], or in $O(m \log (\sigma+\pi)+o c c)$ time by $\operatorname{PSTree}(T)$ [3], where occ is the number of occurrences to report.

In this paper, we propose a new indexing structure for p-matching, the parameterized suffix tray for $T$ (denoted PSTray $(T)$ ). PSTray $(T)$ is a combination of PSTree $(T)$ and $\operatorname{PSA}(T)$ and is an analogue to the suffix tray indexing structure for exact matching [7]. We show that our PSTray $(T)$

(1) occupies $O(n)$ space,

(2) supports PPM queries in $O(m+\log (\sigma+\pi)+o c c)$, and

(3) can be constructed in $O(n)$ time from $\operatorname{PSTree}(T)$ and $\operatorname{PSA}(T)$.

Result (3) implies that PSTray $(T)$ can be constructed in $O(n \min \{\log (\sigma+\pi), \pi\})$ time using $O(n)$ working space [3, 24, 12]. Results (1) and (2) together with this imply that our PSTray $(T)$ is the fastest linear-space indexing structure for PPM which can be built in time linear in $n$.

We emphasize that extending suffix trays for exact matching [7] to parameterized matching is also not straightforward. The suffix tray of a string $T \in \Sigma^{*}$ is a hybrid data structure of the suffix tree and suffix array of $T$, designed as follows: Each of the $O\left(\frac{n}{\sigma}\right)$ carefully-selected nodes of the suffix tree stores an array of fixed size $\sigma$, so that pattern traversals within these selected nodes take $O(m)$ time (this also ensures a total space to be $\left.O\left(\frac{n}{\sigma} \times \sigma\right)=O(n)\right)$. Once the pattern traversal reaches an unselected node, then the search switches to the sub-array of the suffix array of size $O(\sigma)$. This ensures a worst-case $O(m+\log \sigma+o c c)$-time pattern matching with the suffix tray.

Now, recall that the previous-encoded suffixes of $T$ are sequences over an alphabet $\Sigma \cup\{0, \ldots, n-1\}$ of size $\Theta(\sigma+n) \subseteq O(n)$, while the alphabet size of $T$ is $\sigma+\pi$. This means that naïve extensions of suffix trays to PPM would only result in either super-linear $O\left(\frac{n^{2}}{\sigma+\pi}\right)$ space, or $O(m+\log n+o c c)$ query time which can be achieved already with the parameterized suffix array. We overcome this difficulty by using the smallest parameterized encoding (spe) of strings which was previously proposed by the authors in the context of PPM on labeled trees [13], and this leads to our $O(n)$-space parameterized suffix trays with desired $O(m+\log (\sigma+\pi)+o c c)$ query time. 


\section{Preliminaries}

Let $\Sigma$ and $\Pi$ be disjoint ordered sets of characters, respectively called the static alphabet and the parameterized alphabet. We assume that any character in $\Pi$ is lexicographically smaller than any character in $\Sigma$. An element of $(\Sigma \cup \Pi)^{*}$ is called a $p$-string. For a (p-)string $w=x y z, x, y$ and $z$ are called a prefix, substring, and suffix of $w$. The $i$-th character of a (p-)string $w$ is denoted by $w[i]$ for $1 \leq i \leq|w|$, and the substring of a (p-)string $w$ that begins at position $i$ and ends at position $j$ is denoted by $w[i: j]$ for $1 \leq i \leq j \leq|w|$. For convenience, let $w[i: j]=\varepsilon$ if $j<i$. Also, let $w[i:]=w[i:|w|]$ for any $1 \leq i \leq|w|$, and $w[: j]=w[1: j]$ for any $1 \leq j \leq|w|$. For any (p-)string $w$, let $w^{R}$ denote the reversed string of $w$. If a p-string $x$ is lexicographically smaller than a p-string $y$, then we write $x<y$.

Definition 1 (Parameterized match [2]). Two p-strings $x$ and $y$ of the same length are said to parameterized match ( $p$-match) iff there is a bijection $f$ on $\Sigma \cup \Pi$ such that $f(c)=c$ for any $c \in \Sigma$ and $x[i]=f(y[i])$ for any $1 \leq i \leq|x|$.

We write $x \approx y$ iff two p-strings $x, y$ p-match. For instance, if $\Sigma=\{\mathrm{A}, \mathrm{B}\}, \Pi=\{\mathrm{x}, \mathrm{y}, \mathrm{z}\}$, then $X=$ xyzAxxxByzz and $Y=$ zxyAzzzBxyy p-match since there is a bijection $f$ such that $f(\mathrm{~A})=\mathrm{A}, f(\mathrm{~B})=\mathrm{B}, f(\mathrm{x})=\mathrm{z}, f(\mathrm{y})=\mathrm{x}, f(\mathrm{z})=\mathrm{y}$, and

$$
f(\mathrm{x}) f(\mathrm{y}) f(\mathrm{z}) f(\mathrm{~A}) f(\mathrm{x}) f(\mathrm{x}) f(\mathrm{x}) f(\mathrm{~B}) f(\mathrm{y}) f(\mathrm{z}) f(\mathrm{z})=\text { zxyAzzzBxyy }=Y .
$$

Definition 2 (Parameterized Pattern Matching problem(PPM) [2]). Given a text p-string $T$ and a pattern p-string $P$, find all positions $i$ in $T$ such that $T[i: i+|P|-1] \approx p$.

For instance, if $\Sigma=\{\mathrm{A}\}, \Pi=\{\mathrm{x}, \mathrm{y}, \mathrm{z}\}, T=\mathrm{xyzAxxxAyyzAzx}$, and $P=\mathrm{yAzz}$, then the out put for PPM is $\{3,7\}$. We call the positions in the output of PPM the p-beginning positions for given text $T$ and pattern $P$. We say that the pattern $p$-appears in the text $T$ iff the pattern and a substring of the text p-match. In this paper, we suppose that a given text $T$ terminates with a special end-marker $\$$ which occurs nowhere else in $T$. We assume that $\$$ is an element of $\Sigma$ and $\$$ is lexicographically larger than any elements from $\Sigma$ and $\Pi$.

Definition 3 (Previous encoding [2]). For a p-string $w$, the previous encoding $\operatorname{prev}(w)$ is a string of length $|w|$ such that for each $1 \leq i \leq|w|$,

$$
\operatorname{prev}(w)[i]= \begin{cases}w[i] & \text { if } w[i] \in \Sigma, \\ 0 & \text { if } w[i] \in \Pi \text { and } w[j] \neq w[i] \text { for any } 1 \leq j<i, \\ i-j & \text { otherwise, } w[i]=w[j] \text { and } w[i] \neq w[k] \text { for any } j<k<i\end{cases}
$$

Intuitively, when we transform $w$ to $\operatorname{prev}(w)$, the first occurrence of each element of $\Pi$ is replaced with 0 and any other occurrence of the element of $\Pi$ is replaced by the distance to the previous occurrence of the same character, and each element of $\Sigma$ remains the same.

Definition 4 (Smallest parameterized encoding (spe) [13]). For a p-string $w$, the smallest parameterized encoding $\operatorname{spe}(w)$ is the lexicographically smallest p-string such that $w \approx$ $\operatorname{spe}(w)$. 
Namely, spe $(w)$ maps a given string $w$ to the representative of the equivalence class of p-strings under p-matching $\approx$.

For any two p-strings $w_{1}, w_{2}, \operatorname{prev}\left(w_{1}\right)=\operatorname{prev}\left(w_{2}\right) \Leftrightarrow \operatorname{spe}\left(w_{1}\right)=\operatorname{spe}\left(w_{2}\right) \Leftrightarrow w_{1} \approx w_{2}$. For instance, let $\Sigma=\{\mathrm{A}, \mathrm{B}\}, \Pi=\{\mathrm{x}, \mathrm{y}, \mathrm{z}\}, X=$ yxzAyyyBxzz, and $Y=$ zxyAzzzBxyy. Then $\operatorname{prev}(X)=000 \mathrm{~A} 411 \mathrm{~B} 771=\operatorname{prev}(Y)$ and $\operatorname{spe}(X)=\operatorname{xyzAxxxByzz}=\operatorname{spe}(Y)$.

\section{Parameterized suffix trays}

In this section, we propose a new indexing structure called the parameterized suffix tray for PPM, and we discuss its space requirements.

Our parameterized suffix trays are a "hybrid" data structure of parameterized suffix trees and parameterized suffix arrays, which are defined as follows:

Definition 5 (Parameterized suffix trees [3]). The parameterized suffix tree for a p-string $T$, denoted PSTree $(T)$, is a compact trie that stores the set $\{\operatorname{prev}(T[i:])|1 \leq i \leq| T \mid\}$ of the previous encodings of all suffixes of $T$.

See Figure 1 for examples of PSTree $(T)$. We assume that the leaves of $\operatorname{PSTree}(T)$ are sorted in lexicographical order, so that the sequence of the leaves corresponds to the parameterized suffix array for $T$, which is defined below.

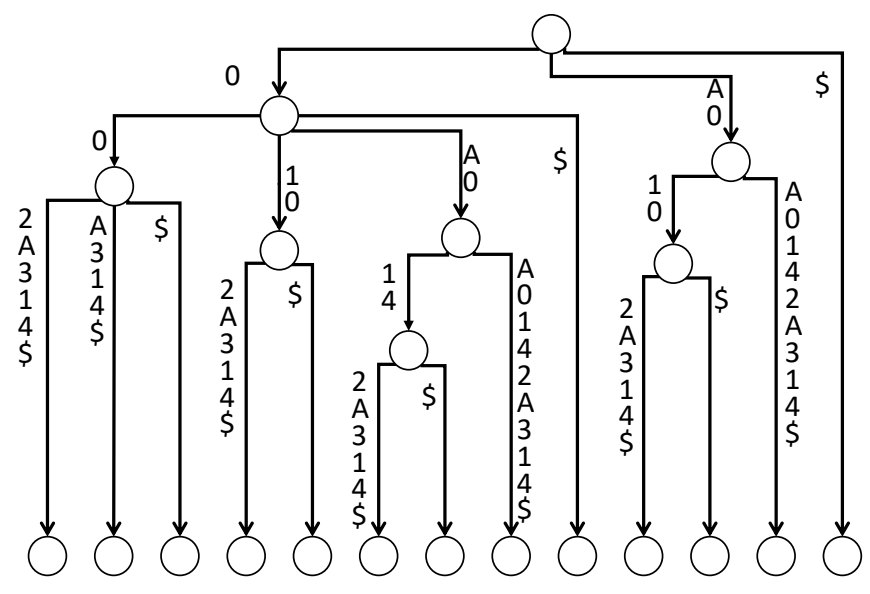

Figure 1: PSTree $(T)$ for a p-string $T=$ zAxAyyxyAxxy, where $\Sigma=\{\mathrm{A}, \$\}, \Pi=\{\mathrm{x}, \mathrm{y}, \mathrm{z}\}$.

Definition 6 (Parameterized suffix arrays [8]). The parameterized suffix array of a pstring $T$, denoted $\operatorname{PSA}(T)$, is an array of integers such that $\operatorname{PSA}(T)[i]=j$ if and only if $\operatorname{prev}(T[j:])$ is the $i$ th lexicographically smallest string in $\{\operatorname{prev}(T[i:])|1 \leq i \leq| T \mid\}$.

Definition 7 (Parameterized longest common prefix arrays [8]). The parameterized longest common prefix array of a p-string $T$, denoted $\operatorname{PLCP}(T)$, is an array of integers such that $\operatorname{PLCP}(T)[1]=0$ and $2 \leq i \leq|T| \operatorname{PLCP}(T)[i]$ stores the length of the longest common prefix between $\operatorname{prev}(T[\operatorname{PSA}(T)[i-1]:])$ and $\operatorname{prev}(T[\operatorname{PSA}(T)[i]:])$. 


\begin{tabular}{|c|c|c|c|}
\hline$i$ & $\overline{\operatorname{PSA}(T)[i]}$ & $T[\operatorname{PSA}(T)[i]:]$ & $\operatorname{PLCP}(i)$ \\
\hline 1 & 6 & $002 \mathrm{~A} 314 \$$ & 0 \\
\hline 2 & 7 & $00 \mathrm{~A} 314 \$$ & 2 \\
\hline 3 & 11 & $00 \$$ & 2 \\
\hline 4 & 5 & $0102 \mathrm{~A} 314 \$$ & 1 \\
\hline 5 & 10 & $010 \$$ & 3 \\
\hline 6 & 3 & 0 A 0142 A $314 \$$ & 1 \\
\hline 7 & 8 & $0 \mathrm{~A} \quad 0144 \$$ & 5 \\
\hline 8 & 1 & 0 A 0 A 0142 A $314 \$$ & 3 \\
\hline 9 & 12 & $0 \$$ & 1 \\
\hline 10 & 4 & A 01102 A $314 \$$ & 0 \\
\hline 11 & 9 & A $010 \$$ & 4 \\
\hline 12 & 2 & A 0 A 0142 A $314 \$$ & 2 \\
\hline 13 & 13 & $\$$ & 0 \\
\hline
\end{tabular}

Figure 2: $\operatorname{PSA}(T)$ and $\operatorname{PLCP}(T)$ for a p-string $T=$ zAxAyyxyAxxy, where $\Sigma=\{\mathrm{A}, \$\}, \Pi=$ $\{\mathrm{x}, \mathrm{y}, \mathrm{z}\}$.

See Figure 2 for examples of $\operatorname{PSA}(T)$ and $\operatorname{PLCP}(T)$.

In addition to the above data structures from the literature, we introduce the following new notions and data structures. For convenience, we will sometimes identify each node of the parameterized suffix tree with the string which is represented by that node.

In what follows, let $\Pi_{T}=\{T[i] \in \Pi|1 \leq i \leq| T \mid\}$ and $\Sigma_{T}=\{T[i] \in \Sigma|1 \leq i \leq| T \mid\}$, namely, $\Pi_{T}$ (resp. $\Sigma_{T}$ ) is the set of distinct characters of $\Pi$ (resp. $\Sigma$ ) that occur in $T$. Let $\pi=\left|\Pi_{T}\right|$ and $\sigma=\left|\Sigma_{T}\right|$.

Definition 8 (P-nodes, branching p-nodes). Let $T$ be a p-string over $\Sigma \cup \Pi$. A node $v$ in $\operatorname{PSTree}(T)$ is called a $p$-node if the number of leaves in the subtree of PSTree $(T)$ rooted at $v$ is at least $\max \{\sigma, \pi\}$. A p-node $v$ is called a branching p-node if at least two children of $v$ in $\operatorname{PSTree}(T)$ are p-nodes.

See Figure 3 for examples of p-nodes and branching p-nodes.

For any $x \in \Pi_{T}$, let $\operatorname{rank}_{T}(x)$ denote the lexicographical rank of $x$ in $\Pi_{T} \cup \Sigma_{T}$. Assuming that $\Pi$ and $\Sigma$ are integer alphabets of polynomial size in $n$, we can compute $\operatorname{rank}_{T}(x)$ for every $x \in \Pi_{T}$ in $O(n)$ time by bucket sort. We will abbreviate $\operatorname{rank}_{T}(x)$ as $\operatorname{rank}(x)$ when it is not confusing.

Definition 9 (P-array). Let $\operatorname{prev}(v)$ be any branching p-node of $\operatorname{PSTree}(T)$, where $v$ is some substring of $T$. The $p$-array $A(\operatorname{prev}(v))$ for $\operatorname{prev}(v)$ is an array of length $\sigma+\pi$ such that for each $x \in \Sigma \cup \Pi, A(\operatorname{prev}(v))[\operatorname{rank}(x)]$ stores a pointer to the child $u$ of $\operatorname{prev}(v)$ such that $\operatorname{prev}(\operatorname{spe}(v) x)$ is a prefix of $u$ if such a child exists, and $A(\operatorname{prev}(v))[\operatorname{rank}(x)]$ stores nil otherwise.

See Figure 4 for an example of a p-array.

Definition 10 (Parameterized suffix tray). The parameterized suffix tray of a p-string $T$, denoted PSTray $(T)$, is a hybrid data structure consisting of $\operatorname{PSA}(T), \operatorname{PLCP}(T)$, and $\operatorname{PSTree}(T)$ where each branching p-node is augmented with the p-array. 


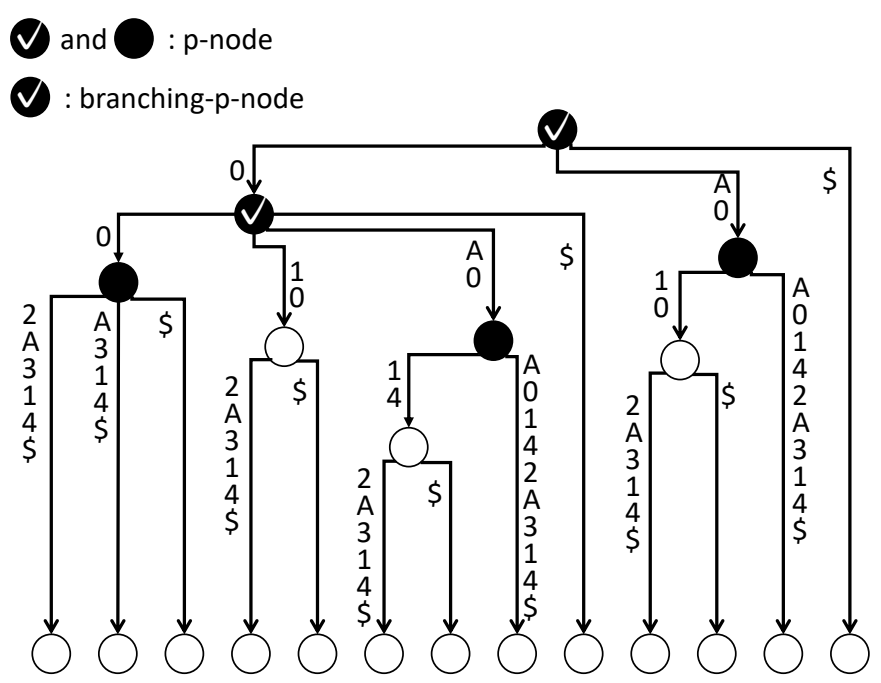

Figure 3: $\operatorname{PSTree}(T)$ for a p-string $T=$ zAxAyyxyAxxy, where $\Sigma=\{\mathrm{A}, \$\}, \Pi=$ $\{\mathrm{x}, \mathrm{y}, \mathrm{z}\}$. Then black nodes are p-nodes because the number of leaves in the subtree of $\operatorname{PSTree}(T)$ rooted at them are at least $\max \{\sigma, \pi\}=3$. Checked nodes are branching p-nodes because at least two children of them in PSTree $(T)$ are p-nodes.

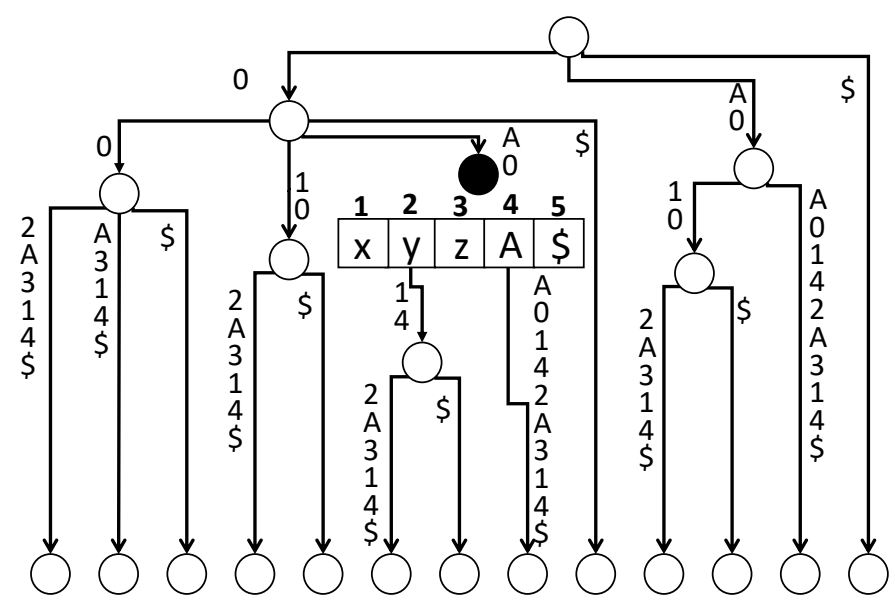

Figure 4: PSTree $(T)$ for a p-string $T=$ zAxAyyxyAxxy, where $\Sigma_{T}=\{\mathrm{A}, \$\}, \Pi_{T}=\{\mathrm{x}, \mathrm{y}, \mathrm{z}\}$. Consider a branching p-node $\operatorname{prev}(v)=\mathrm{OAO}$ where $v$ is e.g. zAx. Then, $A(\mathrm{OAO})[\operatorname{rank}(\mathrm{y})]=$ $A(0 \mathrm{AO})[2]$ stores a pointer to node 0A014 because $\operatorname{spe}(v)=\mathrm{xAy}$ and $\operatorname{prev}(\operatorname{spe}(v) \mathrm{y}=\mathrm{xAyy})=$ $0 \mathrm{~A} 01$ is a prefix of node $0 \mathrm{A014.}$ 
We can show the following lemmas regarding the space requirements of $\operatorname{PSTray}(T)$, by similar arguments to [7] for suffix trays on standard strings.

Lemma 1. For any p-string $T$ of length $n$ over $\Sigma \cup \Pi$, the number of branching $p$-nodes in PSTree $(T)$ is $O\left(\frac{n}{\pi+\sigma}\right)$.

Lemma 2. For any $p$-string $T$ of length $n$, PSTray $(T)$ occupies $O(n)$ space.

\section{$4 \quad$ PPM using parameterized suffix trays}

In this section, we present our algorithm for parameterized pattern matching (PPM) on $\operatorname{PSTray}(T)$. For any node $v$ in $\operatorname{PSTray}(T)$, let $l_{v}=(i, j)$ denote the range of $\operatorname{PSA}(T)$ that $v$ corresponds, namely, $l_{v}=(i, j)$ iff the leftmost and rightmost leaves in the subtree rooted at $v$ correspond to the $i$ th and $j$ th entries of $\operatorname{PSA}(T)$, respectively. For any p-node $v$, we store $l_{v}$ in $v$. Also, for any non-branching p-node $u$, we store a pointer to the unique child of $u$ that is a p-node. These can be easily computed in a total of $O(n)$ time by a standard traversal on PSTree $(T)$.

The basic strategy for PPM with PSTray $(T)$ follows the (exact) pattern matching algorithm with suffix trays on standard strings [7. Namely, we traverse PSTree $(T)$ with a given pattern $P$ from the root, and as soon as we encounter a node that is not a p-node, then we switch to the corresponding range of $\operatorname{PSA}(T)$ and perform a binary search to locate the pattern occurrences. The details follow.

Let $P$ be a pattern p-string of length $m$. We assume that $\Pi$ and $\Sigma$ are disjoint integer alphabets, where $\Pi=\left\{0, \ldots, c_{1} n\right\}$ and $\Sigma=\left\{c_{1} n+1, \ldots, n^{c_{2}}\right\}$ for some positive constants $c_{1}$ and $c_{2}$. Using an array (bucket) $B$ of size $|\Pi|=c_{1} n \in O(n)$, we can compute $\operatorname{prev}(P)$ in $O(m)$ time by scanning $P$ from left to right and keeping the last occurrence of each character $x \in \Pi$ in $P$ in $B[x]$. We can compute spe $(P)$ in $O(m)$ time in a similar manner with a bucket. These buckets are a part of our indexing structure that occupies $O(n)$ total space.

After computing $\operatorname{prev}(P)$ and spe $(P)$, we traverse $\operatorname{prev}(P)$ on $\operatorname{PSTray}(T)$. If $\operatorname{prev}(P[: i])$ for prefix $P[: i](1 \leq i \leq m)$ is represented by a p-node, we can find the out-going edge whose label begins with $\operatorname{prev}(P)[i+1]$ in constant time by accessing the p-array entry $A(\operatorname{prev}(P[: i]))[\operatorname{spe}(P)[i+1]]$. Therefore, we can solve PPM in $O(m+o c c)$ time if $\operatorname{prev}(P)$ is a prefix of some p-node in $\operatorname{PSTray}(T)$. Otherwise (if $\operatorname{prev}(P)$ is not a prefix of any p-node), there exists integer $i$ such that $\operatorname{prev}(P[: i])$ is not a p-node but the parent of $\operatorname{prev}(P[: i])$ is a p-node. In this case, we will use the next lemma.

Lemma 3 (PPM in PSA range (adapted from [8])). Given a pattern p-string $P$ of length $m$ and a range $[j, k]$ in $\mathrm{PSA}(T)$ such that the occ occurrences of $P$ in $T$ lie in the range $[j, k]$ of $\operatorname{PSA}(T)$, we can find them in $O(m+\log (k-j)+$ occ $)$ time by using $\operatorname{PSA}(T)$ and $\operatorname{PLCP}(T)$.

Let $I_{\operatorname{prev}(P[: i])}=(j, k)$ denote the range in $\operatorname{PSA}(T)$ where $\operatorname{prev}(P)$ is a prefix of the suffixes in the range. We apply Lemma 3 to this range so we can find the parameterized occurrences of $P$ in $T$ in $O(m+\log (k-j)+o c c)$ time. By Definition 8 we have $k-j \leq \pi+\sigma$ (recall that $\operatorname{prev}(P[: i])$ is not a p-node). Thus, $O(m+\log (k-j)+o c c) \subseteq O(m+\log (\pi+$ $\sigma)+o c c)$, implying the next theorem. 
Theorem 1. Suppose $|\Pi|=O(n)$. Then, PSTray $(T)$ supports PPM queries in $O(m+$ $\log (\pi+\sigma)+$ occ $)$ time each, where $m$ is the length of a query pattern $P$ and occ is the number of occurrences to report.

\section{Construction of parameterized suffix trays}

Let $T$ be a p-string of length $n$. In this section, we show how to construct PSTray $(T)$ provided that PSTree $(T)$ has already been built. Throughout this section we assume that $\Pi$ and $\Sigma$ are disjoint integer alphabets, both being of polynomial size in $n$, namely, $\Pi=$ $\left\{0, \ldots, n^{c_{1}}\right\}$ and $\Sigma=\left\{n^{c_{1}}+1, \ldots, n^{c_{2}}\right\}$ for some positive constants $c_{1}$ and $c_{2}$. For convenience, we define the following two notions.

Definition 11 (P-function). Let $q, r$ be p-strings such that $q \approx r$. The $p$-function $\mathrm{f}_{q, r}$ : $\Sigma \cup \Pi \rightarrow \Sigma \cup \Pi$ transforms $q$ to $r$, namely, for every $1 \leq i \leq h$

$$
\mathrm{f}_{q, r}(q[i])=r[i]
$$

For instance, if $\Pi=\{\mathrm{x}, \mathrm{y}, \mathrm{z}\}, q=\operatorname{xyxzyyxz} r=\operatorname{zxzyxxzy}$ and $q \approx r$, then $\mathrm{f}_{q, r}(\mathrm{x})=\mathrm{z}$, $\mathrm{f}_{q, r}(\mathrm{y})=\mathrm{x}, \mathrm{f}_{q, r}(\mathrm{z})=\mathrm{y}$ since $q$ can be transformed $r$ by this function.

Definition 12 (F-array). Let $q$ be a p-string and $x \in \Pi_{T}$. The first (left-most) occurrence of $x$ in $q$ is denoted by $i_{q, x}$. The $f$-array of $q$, denoted fpos $(q)$, is an array of length $\pi$ such that $\operatorname{fpos}(q)[\operatorname{rank}(x)]=i_{q, x}$.

For instance, if $\Pi_{T}=\{\mathrm{x}, \mathrm{y}, \mathrm{z}\}$ and $q=\operatorname{xyxzyyxz}$, then $\operatorname{fpos}(q)[\operatorname{rank}(\mathrm{x})]=\mathrm{fpos}(q)[1]=1$, $\operatorname{fpos}(q)[\operatorname{rank}(\mathrm{y})]=\mathrm{fpos}(q)[2]=2$, and $\mathrm{fpos}(q)[\operatorname{rank}(\mathbf{z})]=\mathrm{fpos}(q)[3]$ $=4$.

Given PSTree $(T)$, we show how to construct PSTray $(T)$. It is well known that PSA $(T)$ and $\operatorname{PLCP}(T)$ can be constructed from PSTree $(T)$ in $O(n)$ time. In the following, we consider how to compute $A(\operatorname{prev}(v))$ for every p-node $\operatorname{prev}(v)$ in $\operatorname{PSTree}(T)$.

First, we consider how to compute (branching) p-nodes in PSTree $(T)$. This can be done by a similar method to the suffix tray for exact matching [7], namely:

Lemma 4 (Computing p-node). We can compute all p-nodes and branching p-nodes in $\operatorname{PSTree}(T)$ in $O(n)$ total time.

Our algorithm performs a bottom-up traversal on PSTree $(T)$ and propagates pairs $(\operatorname{fpos}(T[i:]), i)$ from leaves to their ancestors. Each internal p-node $\operatorname{prev}(v)$ will store only a single pair $(\operatorname{fpos}(T[i:]), i)$, where $i$ is the largest position in $T \operatorname{such}$ that $\operatorname{prev}(T[i:])$ is a leaf in the subtree rooted at $\operatorname{prev}(v)$. See also Figure 5 . One can easily compute the pairs for all p-nodes in a total of $O(n)$ time. Then, we compute $\mathrm{f}_{v, \text { spe }(v)}$ for every p-node $\operatorname{prev}(v)$ from the pair $(\operatorname{fpos}(T[i:]), i)$ that is stored in the p-node $\operatorname{prev}(v)$. Finally, for every p-node $\operatorname{prev}(v)$ we compute $A_{\operatorname{prev}(v)}$ from $\mathrm{f}_{v, \operatorname{spe}(v)}$ and $i$.

In what follows, we first show how to compute $A_{\operatorname{prev}(v)}$ from $\mathrm{f}_{v, \operatorname{spe}(v)}$ and $i$ in Lemmas 5 and 6. We then present how to compute $\mathrm{f}_{v, \operatorname{spe}(v)}$ and $i$ from $\operatorname{fpos}(T[i:])$ in Lemma 7, and

\footnotetext{
${ }^{1}$ Indeed, our PSTray $(T)$ construction algorithm works with any position $i$ in the subtree rooted at $\operatorname{prev}(v)$, and we propagate the largest leaf position $i$ to each internal p-node for simplicity.
} 


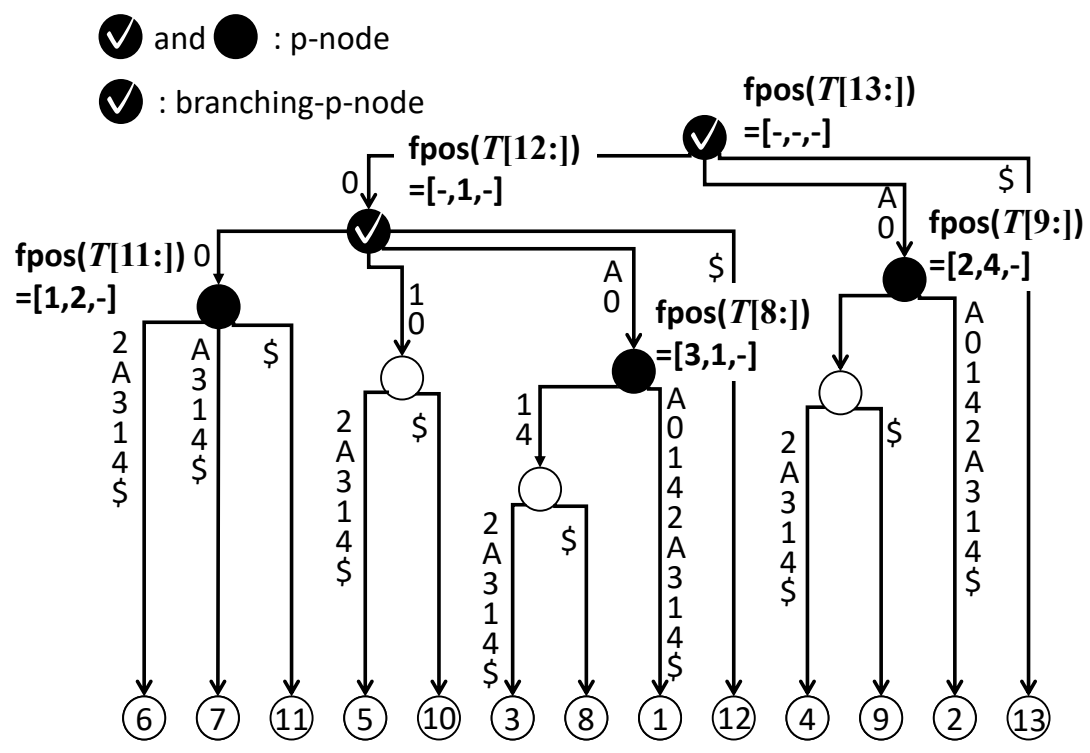

Figure 5: PSTree $(T)$ for a p-string $T=$ zAxAyyxyAxxy, where $\Sigma=\{\mathrm{A}, \$\}$ and $\Pi=\{\mathrm{x}, \mathrm{y}, \mathrm{z}\}$. For instance, we propagate fpos(T[12:]) (coupled with the corresponding position 12) to the p-node 0 .

how to compute $\operatorname{fpos}(T[i:])$ in Lemma 8. These lemmas will ensure the correctness and time complexity of our algorithm.

We consider how to compute $A(\operatorname{prev}(v))$ for a given p-node $\operatorname{prev}(v)$.

Lemma 5. Let $s$ be a p-string. If $\operatorname{prev}(s)[|s|]=k \in\{0, \ldots,|T|-1\}$, then $\operatorname{spe}(s)[|s|]=$ $\operatorname{spe}(s)[|s|-k]$.

Proof. Clear from the definitions of $\operatorname{prev}(\cdot)$ and spe $(\cdot)$.

In the sequel, let $\operatorname{prev}(T[i:])$ be any leaf in the $\operatorname{subtree}$ rooted at $\operatorname{prev}(v)$, where $1 \leq$ $i \leq|T|$. By Lemma 5, we can compute $A(\operatorname{prev}(v))$ if we know spe $(v)[|v|-k+1]$, where $k=\operatorname{prev}(T[i:])[|v|+1]$.

Lemma 6. $\operatorname{spe}(v)[|v|-k+1]=\mathrm{f}_{T[i: i+|v|-1], \operatorname{spe}(T[i: i+|v|-1]) T[i+|v|+k-2]}$.

Proof. Clear from the definitions of $\mathrm{f}_{\text {, }}$, and spe $(\cdot)$.

We can compute $\operatorname{spe}(v)[|v|-k+1]$ if we know $\mathrm{f}_{T[i: i+|v|-1], \operatorname{spe}(v)}$. In the next lemma, we show how to compute $\mathrm{f}_{T[i: i+|v|-1], \operatorname{spe}(v)}$ for all p-nodes $\operatorname{prev}(v)$.

Lemma 7. For every $p$-node $\operatorname{prev}(v)$, we can compute $\mathrm{f}_{T[i: i+|v|-1], \operatorname{spe}(T[i: i+|v|-1])}$ with $\operatorname{prev}(v)=$ $\operatorname{prev}(T[i: i+|v|-1]))$ in amortized $O(\pi)$ time if we know pair $\operatorname{fpos}(T[i:], i)$.

Proof. Let $x_{j}$ be the $j$ th smallest element of $\Pi$ in lexicographically order. Let $y_{l}$ denote the parameterized character in $S=T[i: i+|v|-1]$ such that if $j$ is the left-most occurrence of $y_{l}$ in $S$ (i.e. $j=\min \left\{h \mid S[h]=y_{l}\right\}$ ), then $\left|\Pi_{S[1 . . j]}\right|=l$. For instance, for $S=\mathrm{xAzAyA}$, then $y_{1}=\mathrm{x}, y_{2}=\mathrm{z}$, and $y_{3}=\mathrm{y}$. Let $\left(i_{\operatorname{prev}(v)}, \operatorname{fpos}\left(T\left[i_{\operatorname{prev}(v)}:\right]\right)\right.$ denote the pair stored in 
p-node $\operatorname{prev}(v)$. Consider a set $\mathbf{I}=\left\{\left(i_{\operatorname{prev}(v)}, \operatorname{fpos}\left(T\left[i_{\operatorname{prev}(v)}:\right]\right)[k]\right) \mid \operatorname{prev}(v)\right.$ is a p-node, $1 \leq$ $k \leq \pi\}$ of integer pairs. We sort the elements of $\mathbf{I}$ so that we can compute in $O(1)$ time

$\mathrm{f}_{T[i: i+|v|-1], \operatorname{spe}(v)}\left(y_{l}\right)=x_{l}$ for all p-nodes $\operatorname{prev}(v)$, where $x_{l}$ is the $l$ th smallest parameterized character that occurs in spe $(v)$. We can sort the elements of $\mathbf{I}$ in a total of $O(n)$ time by radix sort, since there are $O\left(\frac{n}{\sigma+\pi}\right)$ p-nodes and each f-array fpos $(T[i:])$ is of length $\pi$. This completes the proof.

We can easily compute $\operatorname{fpos}(T[i:])$ by the following lemma:

Lemma 8. Let $q$ be a p-string. Let $x \in \Pi, y \in \Pi \cup \Sigma$ and $x \neq y$. Then the following equations hold:

$$
\begin{aligned}
\operatorname{fpos}(x q)[\operatorname{rank}(x)] & =1 \\
\operatorname{fpos}(x q)[\operatorname{rank}(y)] & =\operatorname{fpos}(q)[\operatorname{rank}(y)]+1 .
\end{aligned}
$$

Thus we can compute f-arrays $\operatorname{fpos}(T[i:])$ for all $1 \leq i \leq n$ in a total of $O(n)$ time.

Since all the afore-mentioned procedures take $O(n)$ time each, we obtain the main theorem of this section.

Theorem 2. Given a p-string $T$ of length $n$ over alphabet $\Sigma \cup \Pi$ with $\Pi=\left\{0, \ldots, n^{c_{1}}\right\}$ and $\Sigma=\left\{n^{c_{1}}+1, \ldots, n^{c_{2}}\right\}$ for some positive constants $c_{1}$ and $c_{2}$, we can construct PSTray $(T)$ in $O(n)$ time from $\operatorname{PSTree}(T)$.

\section{Conclusions and open questions}

In this paper, we proposed an indexing structure for parameterized pattern matching (PPM) called the parameterized suffix tray PSTray $(T)$, where $T$ is a given text string. Our $\operatorname{PSTray}(T)$ uses $O(n)$ space and supports pattern matching queries in $O(m+\log (\sigma+\pi)+o c c)$ time, where $n=|T|, m$ is the query pattern length, $\sigma$ and $\pi$ are respectively the numbers of distinct static characters and distinct parameterized characters occurring in $T$, and occ is the number of pattern occurrences to report. We also showed how to construct PSTray $(T)$ in $O(n+s(n))$ time, where $s(n)$ denotes the time complexity to build the parameterized suffix tree PSTree $(T)$ for $T$. It is known that $s(n)=\min \{n \pi, n(\log (\pi+\sigma)\}[3,24,12$.

On the other hand, if we use hashing for implementing the branches of the parameterized suffix tree PSTree $(T)$, one can trivially answer PPM queries in $O(m+o c c)$ time with $O(n)$ space. The best linear-space deterministic hashing we are aware of is the one by Ružić [23], which can be built in $O\left(n(\log \log n)^{2}\right)$ time for a set of $n$ keys in the word RAM model with machine word size $\Omega(\log n)$. By associating each node of PSTree $(T)$ with a unique integer (e.g. the pre-order rank), one can regard each branch in PSTree $(T)$ as an integer from the universe of polynomial size in $n$, each fitting in a constant number of machine words. This gives us a deterministic $O\left(n(\log \log n)^{2}+s(n)\right)$-time algorithm for building PSTree $(T)$ with $O(m+o c c)$-time PPM queries. Still, it is not known whether a similar data structure can be build in $O(n+s(n))$ time. We conjecture that our $O(m+\log (\sigma+\pi)+o c c)$ PPM query time would be the best possible for any indexing structure that can be build in $O(n+s(n))$ time. Proving or disproving such a lower bound is an intriguing open problem. 


\section{Acknowledgments}

This work was supported by JSPS KAKENHI Grant Numbers JP18K18002 (YN), JP17H01697 (SI), JP20H04141 (HB), JP18H04098 (MT), and JST PRESTO Grant Number JPMJPR1922 (SI).

\section{References}

[1] U. Baier. Linear-time suffix sorting - A new approach for suffix array construction. In CPM 2016, pages 23:1-23:12, 2016.

[2] B. S. Baker. A theory of parameterized pattern matching: algorithms and applications. In STOC 1993, pages 71-80, 1993.

[3] B. S. Baker. Parameterized pattern matching: Algorithms and applications. J. Comput. Syst. Sci., 52(1):28-42, 1996.

[4] A. Blumer, J. Blumer, D. Haussler, A. Ehrenfeucht, M. T. Chen, and J. Seiferas. The smallest automaton recognizing the subwords of a text. Theoretical Computer Science, 40:31-55, 1985.

[5] A. Blumer, J. Blumer, D. Haussler, R. Mcconnell, and A. Ehrenfeucht. Complete inverted files for efficient text retrieval and analysis. Journal of the ACM, 34(3):578$595,1987$.

[6] M. Burrows and D. J. Wheeler. A block sorting lossless data compression algorithm, 1994.

[7] R. Cole, T. Kopelowitz, and M. Lewenstein. Suffix trays and suffix trists: Structures for faster text indexing. Algorithmica, 72(2):450-466, 2015.

[8] S. Deguchi, F. Higashijima, H. Bannai, S. Inenaga, and M. Takeda. Parameterized suffix arrays for binary strings. In PSC 2008, pages 84-94, 2008.

[9] A. Ehrenfeucht, R. M. McConnell, N. Osheim, and S.-W. Woo. Position heaps: A simple and dynamic text indexing data structure. Journal of Discrete Algorithms, 9(1):100-121, 2011.

[10] M. Farach-Colton, P. Ferragina, and S. Muthukrishnan. On the sorting-complexity of suffix tree construction. J. ACM, 47(6):987-1011, 2000.

[11] N. Fujisato, Y. Nakashima, S. Inenaga, H. Bannai, and M. Takeda. Right-to-left online construction of parameterized position heaps. In PSC 2018, pages 91-102, 2018.

[12] N. Fujisato, Y. Nakashima, S. Inenaga, H. Bannai, and M. Takeda. Direct linear time construction of parameterized suffix and LCP arrays for constant alphabets. In SPIRE 2019, pages 382-391, 2019.

[13] N. Fujisato, Y. Nakashima, S. Inenaga, H. Bannai, and M. Takeda. The parameterized position heap of a trie. In CIAC 2019, pages 237-248, 2019. 
[14] A. Ganguly, R. Shah, and S. V. Thankachan. pBWT: Achieving succinct data structures for parameterized pattern matching and related problems. In SODA 2017, pages 397-407, 2017.

[15] J. Kärkkäinen, P. Sanders, and S. Burkhardt. Linear work suffix array construction. J. ACM, 53(6):918-936, 2006.

[16] D. K. Kim, J. S. Sim, H. Park, and K. Park. Constructing suffix arrays in linear time. J. Discrete Algorithms, 3(2-4):126-142, 2005.

[17] P. Ko and S. Aluru. Space efficient linear time construction of suffix arrays. J. Discrete Algorithms, 3(2-4):143-156, 2005.

[18] G. Kucherov. On-line construction of position heaps. J. Discrete Algorithms, 20:3-11, 2013.

[19] U. Manber and G. Myers. Suffix arrays: A new method for on-line string searches. SIAM J. Computing, 22(5):935-948, 1993.

[20] J. Mendivelso, S. V. Thankachan, and Y. J. Pinzón. A brief history of parameterized matching problems. Discret. Appl. Math., 274:103-115, 2020.

[21] K. Nakashima, N. Fujisato, D. Hendrian, Y. Nakashima, R. Yoshinaka, S. Inenaga, H. Bannai, A. Shinohara, and M. Takeda. DAWGs for parameterized matching: Online construction and related indexing structures. In CPM 2020, pages 26:1-26:14, 2020.

[22] G. Nong, S. Zhang, and W. H. Chan. Two efficient algorithms for linear time suffix array construction. IEEE Trans. Computers, 60(10):1471-1484, 2011.

[23] M. Ružić. Constructing efficient dictionaries in close to sorting time. In ICALP 2008, volume 5125 of Lecture Notes in Computer Science, pages 84-95. Springer, 2008.

[24] T. Shibuya. Generalization of a suffix tree for RNA structural pattern matching. Algorithmica, 39(1):1-19, 2004.

[25] P. Weiner. Linear pattern-matching algorithms. In Proc. of 14th IEEE Ann. Symp. on Switching and Automata Theory, pages 1-11, 1973. 